\title{
含双搌基团大环化合物的合成及其对卤素阴离子检测研究
}

\author{
张读山 陈金平* 曾 毅 于天君 李 嫕* \\ ( ${ }^{a}$ 中国科学院理化技术研究所 光化学转换与功能材料重点实验室 北京 100190)
}

\begin{abstract}
摘要 通过片段偶联法方便地合成了骨架上含有两个脲基团的 O-杂大环化合物骨架 cycle 1, 并对 cycle 1 进一步修饰 得到了化合物 cycle 2 和 cycle 3. 紫外吸收光谱滴定实验表明, 二氯甲烷中 cycle 2 和 cycle 3 可与卤素阴离子 1: 1 结合, 结合常数为 $10^{5} \sim 10^{8} \mathrm{~L} \cdot \mathrm{mol}^{-1}$, 核磁滴定实验证实环上䐂基团是阴离子与大环结合的主要位点.

关键词 大环化合物; 脉; 卤素阴离子响应; 吸收
\end{abstract}

\section{Bisurea-Functionalized Macrocycles: Synthesis and Halide Anion-Response}

\author{
Zhang, Dushan Chen, Jinping* Zeng, Yi Yu, Tianjun Li, $\mathrm{Yi}^{*}$ \\ (Key Laboratory of Photochemical Conversion and Optoelectronic Materials, Technical Institute of Physics and Chemistry, \\ Chinese Academy of Sciences, Beijing 100190)
}

\begin{abstract}
Oxo-bisurea-functionalized macrocycles, cycle 1, cycle 2, and cycle 3, were synthesized by a stepwise coupling approach. Absorption studies reveal that cycle 2 and cycle 3 can bind with halide anions in a $1: 1$ stoichiometry with binding constants of $10^{5} \sim 10^{8} \mathrm{~L} \cdot \mathrm{mol}^{-1}$. ${ }^{1} \mathrm{H}$ NMR titration experiments show that urea groups are the binding sites and play important roles in the binding strength.

Keywords macrocycle; urea; halide anion-responsive; absorption
\end{abstract}

大环类化合物因其独特的化学结构和性质已广泛 应用于分子/离子识别 ${ }^{[1]}$ 、分子自组装 ${ }^{[2]}$ 以及主客体化 学 ${ }^{[3]}$ 等研究领域, 在药物、新材料研发以及和生命科学 等方面也有着重要的意义, 设计合成具有特定结构和功 能的大环化合物一直是合成有机化学中的重要研究课 题. 脲基团是一种理想的阴离子受体, 可以和多种阴离 子发生相互作用 ${ }^{[4]}$, 将脲基团引入到大环化合物结构中, 有望得到对阴离子具有良好识别功能的大环化合物. 目 前, 文献报道的含嫝基团的大环化合物合成方法通常合 成步骤较长, 产率低 ${ }^{[5]}$, 虽然采用二异氧酸酯和二氨基 化合物的反应合成含䣝基团大环化合物可以大大提高 成环产率 ${ }^{[6]}$, 但是原料二异氰酸酯本身具有较强的毒性, 制备通常需要使用剧毒的光气为原料. 本工作利用羰基 二咪唑和氨基一步反应合成腿基团，通过片段偶联法方 便地合成了一类对卤素阴离子响应的含双脲基团的大
环化合物, 并通过吸收光谱和核磁共振方法研究了它们 与卤素阴离子的相互作用.

\section{1 实验部分}

\section{1 仪器及试剂}

Varian Excalibur 3100 红外光谱仪; Bruker Avance П-400 核磁共振仪; Waters GCT Premier 高分辨液质联 用仪; XT4-100X 显微熔点仪; Shimadzu UV-1601PC 紫 外-可见吸收光谱仪. 所用原料和试剂如无说明均为北 京化学试剂公司普通分析纯或化学纯试剂; 合成用丙酮 经无水碳酸钾干燥后重蒸; 四氢呋喃(THF)用金属钠干 燥回流后重蒸; 吸收光谱测试用 $\mathrm{CH}_{2} \mathrm{Cl}_{2}$ 为迪马公司色 谱纯试剂, 羰基二咪唑(CDI)为上海共价化学科技有限 公司化学纯产品.

\footnotetext{
*E-mail: yili@mail.ipc.ac.cn; chenjp@mail.ipc.ac.cn

Received September 5, 2012; revised September 27, 2012; published online October 11, 2012.

Project supported by the National Natural Science Foundation of China (Nos. 21233011, 21172229, 21002109, 21103210), the National Basic Research Program (No. 2010CB934500) and the Beijing Nova Program.

国家自然科学基金(Nos. 21233011, 21172229, 21002109, 21103210)、国家重点基础研究发展规划(No. 2010CB934500)和北京市科技新星计划资助项目.
} 


\section{2 含脲基大环化合物骨架的合成}

\subsubsection{1,3-二(3-着基苯基)䐂(1)的合成}

在 $50 \mathrm{~mL}$ 的两口瓶中将间氨基酚 $2.18 \mathrm{~g}(20 \mathrm{mmol})$ 和羰基二咪唑(CDI) $1.62 \mathrm{~g}$ (10 mmol)溶于 $40 \mathrm{~mL}$ THF 中, 加热回流约 $4 \mathrm{~h}$, 反应完毕后, 将 THF 旋干, 剩余物 溶于乙酸乙酯，用水、稀盐酸洗涤除去没有反应的间氨 基酚和生成的咪坐, 无水硫酸镁干燥, 过滤浓缩, 柱层 析分离(洗脱剂为乙酸乙酯)得到 $2 \mathrm{~g}$ 白色固体, 产率 82\%. m.p. 217 $218{ }^{\circ} \mathrm{C} ;{ }^{1} \mathrm{H}$ NMR (400 MHz, DMSO-d $d_{6}$ ) $\delta: 9.30(\mathrm{~s}, \mathrm{ArOH}, 2 \mathrm{H}), 8.47(\mathrm{~s}, \mathrm{CONH}, 2 \mathrm{H}), 7.00 \sim 7.04$ (m, ArH, 4H), $6.76(\mathrm{dd}, J=8.1,1 \mathrm{~Hz}, \mathrm{ArH}, 2 \mathrm{H}), 6.35$ (dd, $J=8.0,1.9 \mathrm{~Hz}, \mathrm{ArH}, 2 \mathrm{H})$.

1.2.2 1,3-二[3-(4,6-二氯-1,3,5-三嗪-2-羟基)苯基]艮 (2) 的合成

在 $50 \mathrm{~mL}$ 的两口瓶中将 $5.5 \mathrm{~g}(30 \mathrm{mmol})$ 三聚氯氰溶 于 $70 \mathrm{~mL}$ 的 $\mathrm{THF}$ ，将反应体系置于冰浴之中，将 $2.4 \mathrm{~g}$ (10 mmol) (1)和 $7 \mathrm{~mL}$ (40 mmol)的二异丙基乙基胺 (DIPEA)溶于 $30 \mathrm{~mL}$ THF, 将此混合液滴加到两口瓶中, 约 $1 \mathrm{~h}$ 滴加完毕. 通过 TLC 板检测反应, 待反应完毕后, 停止反应, 直接通过短的减压硅胶柱除去铵盐和部分未 反应的三聚氯氰, 将滤液在低温下旋干, 剩余物溶于乙 酸乙酯, 分别用饱和食盐水、稀盐酸、水洗, 无水硫酸 镁干燥, 得到粗产品, 然后再将粗产品在 $[V($ 氯仿 $)$ : $V($ 乙酸乙酯 $)=1:$ 1] 中重结晶得到 $23.4 \mathrm{~g}$ 白色固体, 产 率为 $62 \%$ m.p. $204 \sim 206{ }^{\circ} \mathrm{C} ;{ }^{1} \mathrm{H} \mathrm{NMR}(400 \mathrm{MHz}$, DMSO- $\left.d_{6}\right) \delta: 9.45$ (s, CONH, 2H), 7.50 (t, $J=2 \mathrm{~Hz}, \mathrm{ArH}$, 2H), 7.34 (t, $J=8.1 \mathrm{~Hz}, \operatorname{ArH}, 2 \mathrm{H}), 7.26(\mathrm{~d}, J=9.1 \mathrm{~Hz}$, ArH, 2H), $6.86(\mathrm{dd}, J=8.0,1.6 \mathrm{~Hz}, \mathrm{ArH}, 2 \mathrm{H})$; MS-ESI $m / z: 538.95[\mathrm{M}-\mathrm{H}]^{-}, 574.93[\mathrm{M}+\mathrm{Cl}]^{-}, 601.95[\mathrm{M}+$ $\left.\mathrm{NO}_{3}\right]^{-}$.

\subsubsection{Cycle 1 的合成}

分别将 $1.08 \mathrm{~g}(2 \mathrm{mmol}) 2$ 和 $0.49 \mathrm{~g}(2 \mathrm{mmol}) 1$ 溶于 $120 \mathrm{~mL}$ 丙酮中, 室温下同时向 $7.5 \mathrm{~mL}$ (8 mmol) DIPEA 的 $560 \mathrm{~mL}$ 丙酮溶液中滴加, 约 $8 \mathrm{~h}$ 滴加完毕, 置于室温 反应 $3 \mathrm{~d}$. TLC 检测反应, 待反应完毕后将丙酮旋干, 通 过柱层析分离 [洗脱剂为 $V$ (乙酸乙酯) $: V(\mathrm{THF})=2: 1$ ] 得到白色固体 $0.77 \mathrm{~g}$, 产率为 $54 \%$. m.p. $>300{ }^{\circ} \mathrm{C} ;{ }^{1} \mathrm{H}$

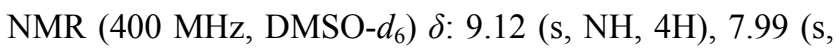
$\mathrm{ArH}, 4 \mathrm{H}), 7.38$ (t, $J=8.1 \mathrm{~Hz}, \mathrm{ArH}, 4 \mathrm{H}), 6.86 \sim 6.91(\mathrm{~m}$, $\mathrm{ArH}, 8 \mathrm{H})$; IR (KBr) v: 3289, 3132, 1689, 1648, 1614, 1541, $1238 \mathrm{~cm}^{-1}$; HRMS-ESI-TOF calcd for $\mathrm{C}_{32} \mathrm{H}_{19} \mathrm{Cl}_{2}$ $\mathrm{N}_{10} \mathrm{O}_{6} \quad[\mathrm{M}-\mathrm{H}]^{-}:$709.0866, found 709.0859.

\section{3 含腿基大环化合物的进一步修饰}

\subsubsection{Cycle 2 的合成}

在 $50 \mathrm{~mL}$ 的两口瓶中将 $50 \mathrm{mg}(0.07 \mathrm{mmol})$ cycle 1,
$169 \mathrm{mg}(0.7 \mathrm{mmol})$ 二辛胺和 $90 \mathrm{mg}(0.7 \mathrm{mmol})$ 的 DIPEA 溶于 $10 \mathrm{~mL}$ 干燥的 THF 中. 通氮气除氧 $15 \mathrm{~min}$, 然后加 热回流 $3 \mathrm{~h}$. 待反应完毕后冷却至室温, 减压除去溶剂, 将剩余物溶于乙酸乙酯, 依次用稀盐酸 $\left(0.2 \mathrm{~mol} \cdot \mathrm{L}^{-1}\right)$ 、饱 和食盐水、水洗涤, 有机层用无水硫酸镁干燥, 粗产品 通过柱层析分离 [洗脱剂为 $V$ (乙酸乙酯)： $V$ (二氯甲 烷 $)=2: 1$ 得到白色固体 $70 \mathrm{mg}$, 产率为 $90 \%$. m.p. $227 \sim 230{ }^{\circ} \mathrm{C} ;{ }^{1} \mathrm{H}$ NMR (400 MHz, DMSO- $\left.d_{6}\right) \delta: 8.84(\mathrm{~s}$, $\mathrm{NH}, 4 \mathrm{H}), 8.00(\mathrm{t}, J=2.1 \mathrm{~Hz}, \mathrm{ArH}, 4 \mathrm{H}), 7.28(\mathrm{t}, J=8.1 \mathrm{~Hz}$, ArH, 4H), $6.71 \sim 6.75(\mathrm{~m}, \mathrm{ArH}, 4 \mathrm{H}), 3.15(\mathrm{t}, J=6.1 \mathrm{~Hz}$, $\left.\mathrm{NCH}_{2}, 8 \mathrm{H}\right), 0.82 \sim 1.21\left(\mathrm{~m}, \mathrm{CH}_{2}, 48 \mathrm{H}\right), 0.81(\mathrm{t}, J=6.9 \mathrm{~Hz}$, $\left.\mathrm{CH}_{3}, 12 \mathrm{H}\right)$; IR (KBr) v: 3307, 2923, 2852, 1644, 1587, 1567, 1427, $748 \mathrm{~cm}^{-1}$; HRMS-ESI-TOF calcd for $\mathrm{C}_{64} \mathrm{H}_{88} \mathrm{~N}_{12} \mathrm{OCl}_{6}$ : $[\mathrm{M}+\mathrm{Cl}]^{-}$1155.6638, found 1155.6656 .

\subsubsection{Cycle 3 的合成}

在 $50 \mathrm{~mL}$ 的两口瓶中将 $0.32 \mathrm{~g}(0.45 \mathrm{mmol})$ cycle 1, $0.68 \mathrm{~g}$ (1 mmol) 3,4,5-三-十二烷氧基苯甲胺和 $0.52 \mathrm{~g} \mathrm{(4}$ mmol) DIPEA 溶于 $25 \mathrm{~mL}$ 干燥的四氢呋喃, 氮气氛围下 加热回流 $2 \mathrm{~h}$. 待反应完毕后冷却至室温, 减压除去溶 剂, 将剩余物溶于乙酸乙酯, 依次用稀盐酸 $(0.2 \mathrm{~mol}$ $\left.\mathrm{L}^{-1}\right)$ 、饱和食盐水、水洗涤, 有机层分离并用无水硫酸 镁干燥, 粗产品通过柱层析分离(洗脱剂为乙酸乙酯)得 到白色固体 $0.52 \mathrm{~g}$, 产率 $60 \%$. m.p. $190 \sim 194{ }^{\circ} \mathrm{C} ;{ }^{1} \mathrm{H}$ NMR $\left[400 \mathrm{MHz}, V\left(\mathrm{CDCl}_{3}\right): V\left(\mathrm{CD}_{3} \mathrm{OD}\right)=5: 1\right] \delta$ : $7.91 \sim 8.00(\mathrm{~m}, \mathrm{ArH}, 4 \mathrm{H}), 7.15 \sim 7.21(\mathrm{~m}, \mathrm{ArH}, 4 \mathrm{H})$, $6.70 \sim 6.76(\mathrm{~m}, \mathrm{ArH}, 8 \mathrm{H}), 6.32(\mathrm{~d}, \mathrm{ArH}, 4 \mathrm{H}), 4.26(\mathrm{~d}, J=$ $\left.15.0 \mathrm{~Hz}, \mathrm{ArCH}_{2}, 4 \mathrm{H}\right), 3.77 \sim 3.85\left(\mathrm{~m}, \mathrm{OCH}_{2}, 12 \mathrm{H}\right), 1.66 \sim$ 1.70 (m, $\left.\mathrm{OCH}_{2} \mathrm{CH}_{2}, 12 \mathrm{H}\right), 1.37$ (bs, $\mathrm{OCH}_{2} \mathrm{CH}_{2} \mathrm{CH}_{2}, 12 \mathrm{H}$ ), 1.20 (br, $\mathrm{CH}_{2}, 96 \mathrm{H}$ ), 0.82 (t, $J=6.5 \mathrm{~Hz}, \mathrm{CH}_{3}, 18 \mathrm{H}$ ); IR (KBr) $v: 2923,2853,1645,1591,1436,751 \mathrm{~cm}^{-1}$; HRMS-ESI-TOF calcd for $\mathrm{C}_{118} \mathrm{H}_{180} \mathrm{~N}_{12} \mathrm{O}_{12}\left[\mathrm{M}+\mathrm{HCO}_{2}\right]^{-}$: 2002.3820, found 2002.6385 $\left[\mathrm{M}+\mathrm{HCO}_{2}\right]^{-}$(without calibration).

\section{2 结果与讨论}

\section{1 含䐖基大环化合物骨架的合成与表征}

本工作选择羰基二咪唑和氨基通过一步反应合成 嫝基团，反应步骤简单，产率高; 以三聚氯㲵作为成环 的连接单元, 利用三聚氯氰上 3 个氯原子活性的不同 ${ }^{[7]}$, 通过控制反应温度使取代反应分步进行, 实现利用片段 偶联法合成目标大环化合物. 具体合成路线如 Scheme 1 所示，首先以大大过量的间氨基酚与羰基二咪唑(CDI) 在四氢呋喃中回流，间氨基酚中的氨基与 CDI 缩合得到 含有嫝基团的片段 $\mathbf{1}$, 产率为 $82 \%$; 片段 1 进一步与连 接单元三聚氯氯在冰水浴下 $\left(0{ }^{\circ} \mathrm{C}\right)$ 反应, 高选择性地得 
到只取代一个氯原子的片段 2 , 产率为 $62 \%$; 片段 2 和 片段 1 室温下在二异丙基乙基胺存在下，偶联得到含有 双脲基团的大环骨架 cycle 1, 反应成环产率达到 54\%. Cycle 1 环状结构的形成首先通过核磁和高分辨质谱测 试得到证实, 核磁共振谱图显示为简单的四组峰, 分别 对应活性脲基团上的质子峰( $\delta$ 9.12)和苯环上的三组氢, 表明形成的结构是完全对称的. 高分辨质谱得到质谱信 号为 709.0866, 与 cycle 1 结构理论计算值 709.0859 $[\mathrm{M}-\mathrm{H}]^{-}$完全吻合.

为了进一步证实反应中确实形成了环状结构, 而不 是链状寡聚物, 我们通过向产物的四氢呋喃溶液中挥发 甲醇得到其单晶, $\mathrm{X}$ 射线单晶衍射法得到了化合物的单 晶结构, 如图 1 所示. 单晶结构明确表明所得产物 cycle 1 为环状化合物, 晶体中 cycle 1 骨架形成的空腔呈平行 四边形的结构, 其两条长度边分别为 9.3 和 $9.9 \AA$, 两个 三嗪环分别位于环平面的两侧呈 1,3 交替构型, 两个嫝 基团均朝向环的内侧, 苯环和脲基团之间的扭转的角度 为 $19.7^{\circ}$, 在其堆积结构中(溶剂分子为甲醇), 两个三嗪
环呈反平行排列，一个分子中三嗪环上的氯原子位于另 一个分子三嗪环平面的垂直方向，与三嗪环之间的距离 为 $3.4 \AA$.

\section{2 含䐖基大环化合物骨架的进一步修饰}

大环骨架上的氯原子为分子进一步修饰和功能化 提供了条件，含多个烷基长链的氨基化合物可与三嗪环 上第三个氯原子进行亲和取代反应，得到多个烷基长链 修饰的大环化合物，这种修饰一方面增加了大环化合物 在有机溶剂中的溶解性, 另一方面也为其功能化, 如在 凝胶、液晶等方面的性质研究提供了可能. 本工作中在 DIPEA 催化下分别以二辛胺和 3,4,5-三一十二烷氧基苯 甲胺为原料，通过亲核取代反应得到了 cycle 2 和 cycle 3, 产率分别为 $90 \%$ 和 $60 \%$, 具体路线如 Scheme 2 所示. cycle 2 合成产率比 cycle 3 高, 推测可能是因为仲胺 (二 辛胺)的亲核性强于伯胺, 与三聚氯氰环中第 3 个氯原 子的反应活性更高所致.<smiles>Nc1cccc(O)c1</smiles><smiles>O=C(Nc1cccc(O)c1)Nc1cccc(O)c1</smiles><smiles>CC(C)(C)C(C)(C)C(C)(C)C</smiles>

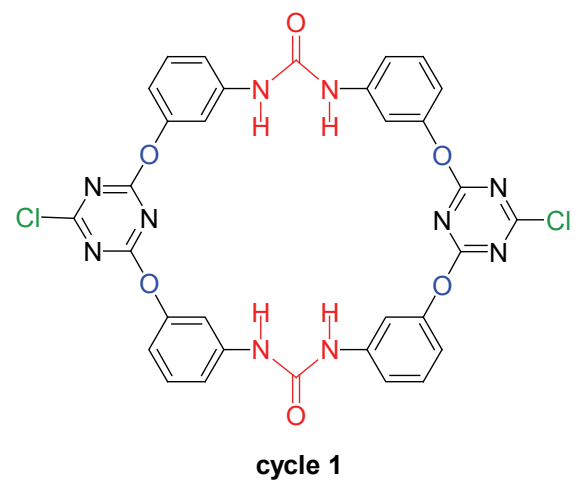

Scheme 1
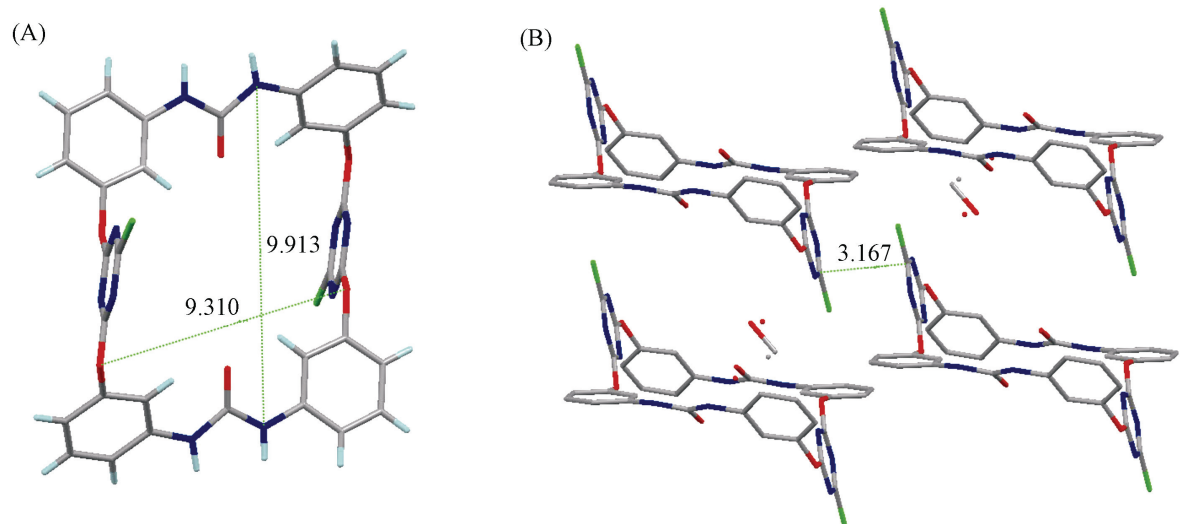

图 1 cycle 1 晶体结构图

Figure 1 Single crystal structure of the cycle 1

(A) Top view, (B) packing style and side view 


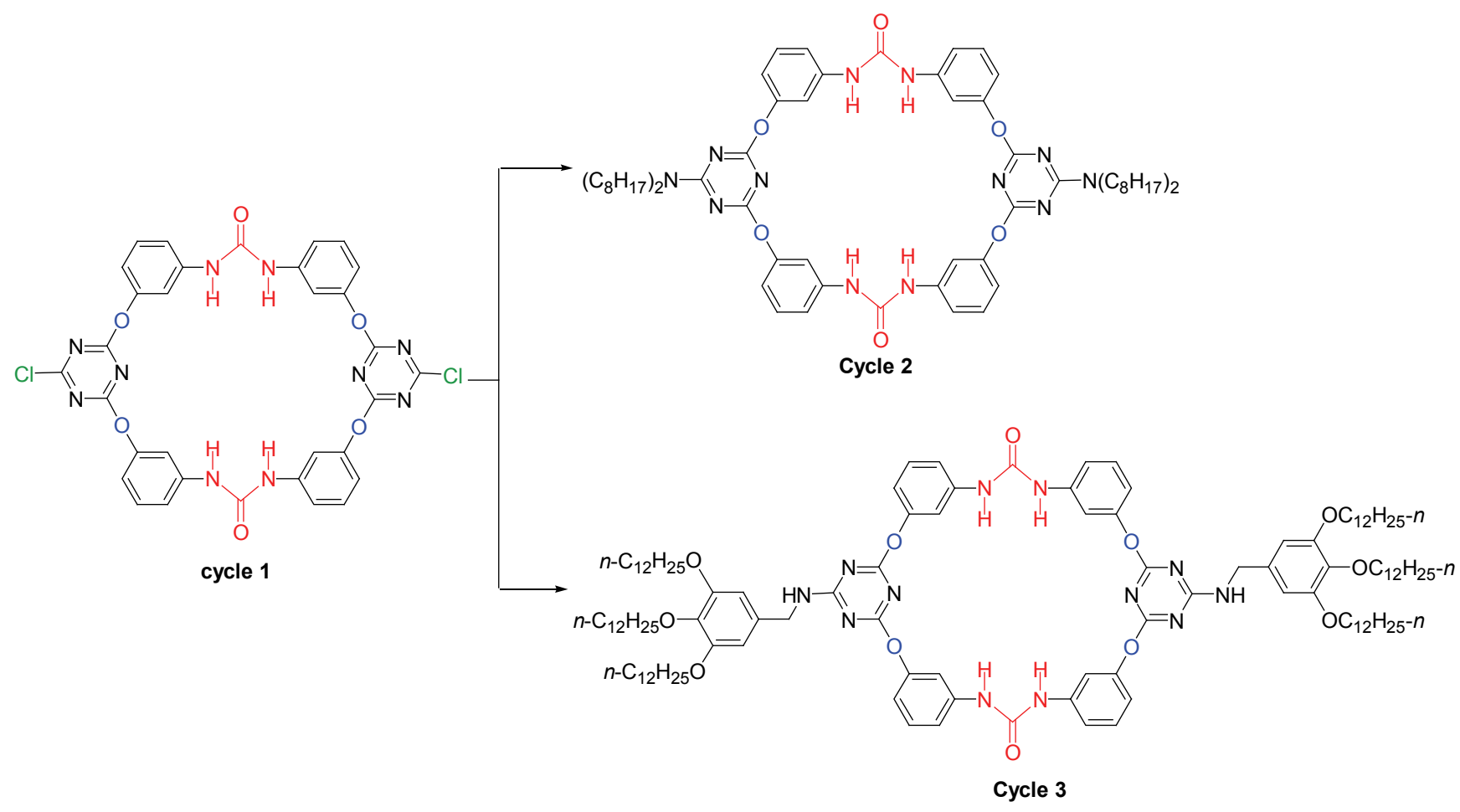

Scheme 2

\section{3 含脲基团大环化合物与卤素阴离子相互作用}

利用吸收光谱滴定的方法研究了大环化合物 cycle 2 和 cycle 3 与卤素阴离子的相互作用, 图 $2 \mathrm{~A}$ 为 cycle 2 (浓度 $\left.2 \times 10^{-5} \mathrm{~mol} \cdot \mathrm{L}^{-1}\right)$ 的吸收光谱随 $\mathrm{Cl}^{-}$离子 $\left(n-\mathrm{Bu}_{4} \mathrm{~N}-\right.$ $\mathrm{Cl}$ 溶液)浓度的变化图, 随着 $\mathrm{Cl}^{-}$离子的加入, cycle 2 在 $239 \mathrm{~nm}$ 处的吸收带略微下降并伴随蓝移, 而 $255 \mathrm{~nm}$ 处 的肩峰逐渐增强并红移, 最终在 $260 \mathrm{~nm}$ 处形成了一个 强的吸收峰, 表明 $\mathrm{Cl}^{-}$离子的加入对大环化合物的吸收 光谱有显著影响, 等吸收点 (235 和 $252 \mathrm{~nm}$ )的出现表明 此平衡体系中只含有两个物种. 以 $260 \mathrm{~nm}$ 处的吸光度 对 $\mathrm{Cl}^{-}$离子浓度作图, 得到图 $2 \mathrm{~A}$ 插入图, 当 $\left[\mathrm{Cl}^{-}\right] /[\mathrm{cycle}$ 2] $=1 / 1$ 时, 体系吸光度达到最大, 继续加入 $\mathrm{Cl}^{-}$离子, cycle 2 的吸收光谱不再变化, 表明 cycle 2 与 $\mathrm{Cl}^{-}$离子的 结合模型为 $1: 1$, 亦即一个大环化合物分子与一个 $\mathrm{Cl}^{-}$ 离子结合, 通过非线性拟合得到其结合常数为 $7.4 \times 10^{7}$ $\mathrm{L} \cdot \mathrm{mol}^{-1}$. $\mathrm{Cl}^{-}$离子对 cycle 3 的吸收光谱滴定实验结果与 cycle 2 相似(图 2B), 研究结果表明, cycle 3 与 $\mathrm{Cl}^{-}$离子也

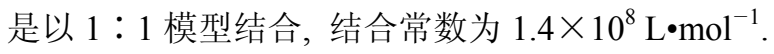

利用紫外吸收光谱滴定实验进一步研究了 cycle 2 和 cycle 3 与其它卤素阴离子 $\mathrm{F}^{-}, \mathrm{Br}^{-}, \mathrm{I}^{-}$离子的相互作 用. 与 $\mathrm{Cl}^{-}$离子滴定吸收光谱变化类似, 随着 $\mathrm{F}^{-}, \mathrm{Br}^{-}, \mathrm{I}^{-}$ 阴离子的加入, 吸收光谱在 $239 \mathrm{~nm}$ 处的吸收带略微下 降, 而在 $260 \mathrm{~nm}$ 处出现一个新的吸收峰, 在 235 和 252 $\mathrm{nm}$ 处出现等吸收点. 以 $260 \mathrm{~nm}$ 处的吸光度对阴离子浓 度作图, 非线性拟合结果表明, cycle 2 和 cycle 3 与不同
卤素阴离子的结合比均为 $1: 1$, 得到大环化合物与卤 素阴离子的结合常数, 结果列于表 1 中. 从结合常数可 以看出, 大环化合物与卤族元素阴离子 $\left(\mathrm{F}^{-}, \mathrm{Cl}^{-}, \mathrm{Br}^{-}\right.$, $\left.\mathrm{I}^{-}\right)$的结合常数随原子序数的增大而减小, 二氯甲烷中 与 $\mathrm{F}^{-}$离子的结合常数比 $\mathrm{I}^{-}$离子高两个数量级以上. 分析 是因为腿基团与卤素离子间的相互作用为氢键, 随着卤 素离子原子序数的增大, 其离子半径增大, 离子的电荷 密度降低, 碱性减弱, 卤素离子与嫝基团之间的氢键相 互作用减弱, 结合常数降低 ${ }^{[8]}$. cycle 2 和 cycle 3 与同一 阴离子结合常数略有差异, 可能是因为不同取代基修饰 的大环在溶液中的溶剂化作用和构象不同所致.

表 1 室温 cycle 2 和 cycle 3 在 $\mathrm{CH}_{2} \mathrm{Cl}_{2}$ 中与卤素阴离子的结合 常数 $\left[K_{\mathrm{a}} /\left(\mathrm{L} \cdot \mathrm{mol}^{-1}\right)\right]$

Table 1 Association constants $\left[K_{\mathrm{a}} /\left(\mathrm{L} \cdot \mathrm{mol}^{-1}\right)\right]$ of cycle 2 and cycle 3 binding with halide anions in $\mathrm{CH}_{2} \mathrm{Cl}_{2}$ at room temprature

\begin{tabular}{ccccc}
\hline 阴离子 & $\mathrm{F}^{-}$ & $\mathrm{Cl}^{-}$ & $\mathrm{Br}^{-}$ & $\mathrm{I}^{-}$ \\
\hline cycle 2 & $2.1 \times 10^{8}$ & $7.4 \times 10^{7}$ & $4.8 \times 10^{6}$ & $3.5 \times 10^{5}$ \\
cycle 3 & $3.1 \times 10^{8}$ & $1.4 \times 10^{8}$ & $1.4 \times 10^{7}$ & $2.3 \times 10^{6}$ \\
\hline
\end{tabular}

利用 ${ }^{1} \mathrm{H}$ NMR 滴定实验研究了大环化合物与卤素 阴离子的结合位点. 为了能够清楚地观察到活性氢(腿 基团 $\mathrm{NH}$ )核磁信号的变化, 选择氝代DMSO 作为核磁滴 定实验的溶剂. 图 3 为随体系中加入不同物质的量 $\mathrm{Cl}^{-}$ 后 cycle 2 的 ${ }^{1} \mathrm{H}$ NMR 图谱, 结构式中 $\mathrm{H}_{\mathrm{a}}, \mathrm{H}_{\mathrm{c}}, \mathrm{H}_{\mathrm{e}}$ 分别归 属于核磁信号峰 $\mathrm{a}, \mathrm{c}$ 和 $\mathrm{e}, \mathrm{H}_{\mathrm{b}}$ 和 $\mathrm{H}_{\mathrm{d}}$ 由于化学位移相近, 为 $\delta 6.7$ 左右的两个双重峰, 没有加入 $\mathrm{Cl}^{-}$时, 环上脲基 

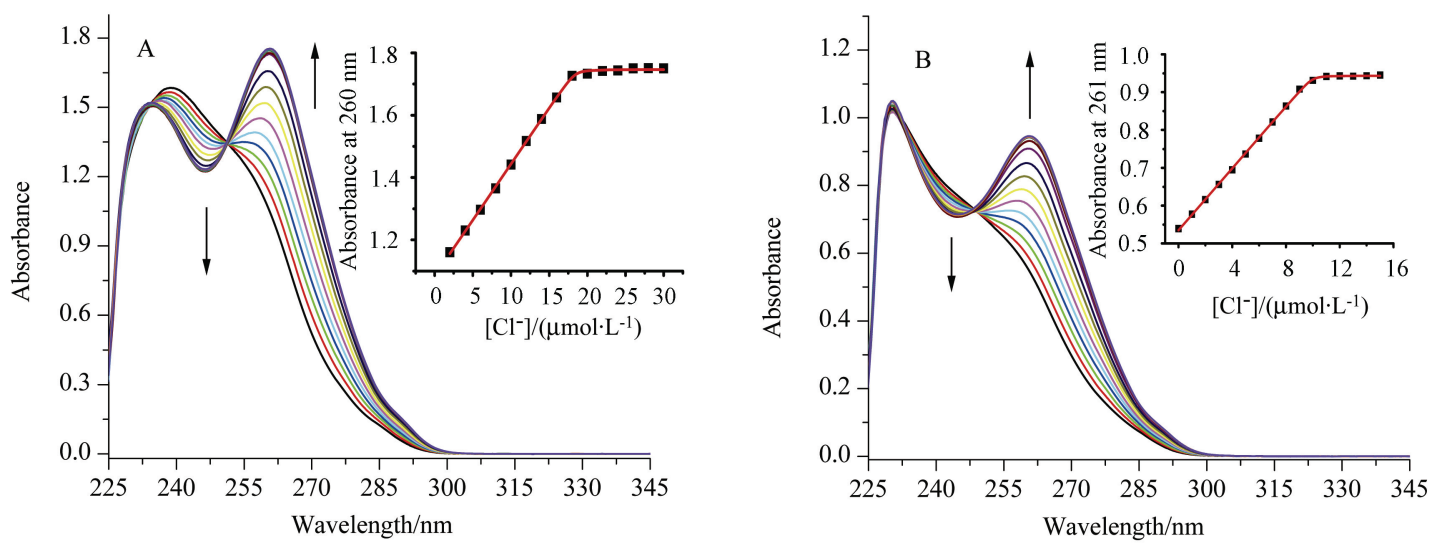

图 2 cycle 2(图 A)和 cycle 3(图 B) 在二氯甲烷中的吸收光谱随 $\mathrm{Cl}^{-}$浓度变化图 ([cycle 2] $=2 \times 10^{-5} \mathrm{~mol} \cdot \mathrm{L}^{-1}$, [cycle 3] $=1 \times 10^{-5}$ $\mathrm{mol} \cdot \mathrm{L}^{-1}, n-\mathrm{Bu}_{4} \mathrm{NCl}$ 的浓度为 $\left.0 \sim 3 \times 10^{-5} \mathrm{~mol} \cdot \mathrm{L}^{-1}\right)$

Figure 2 Absorption spectra of cycle $2\left(2 \times 10^{-5} \mathrm{~mol} \cdot \mathrm{L}^{-1}\right)(\mathrm{A})$ and cycle $3\left(1 \times 10^{-5} \mathrm{~mol} \cdot \mathrm{L}^{-1}\right)$ (B) upon titration with $n-\mathrm{Bu}_{4} \mathrm{NCl}(0 \sim$ $\left.3 \times 10^{-5} \mathrm{~mol} \cdot \mathrm{L}^{-1}\right)$ in $\mathrm{CH}_{2} \mathrm{Cl}_{2}$

Inset: plots of absorbance at 260 or $261 \mathrm{~nm}$ vs. $\left[\mathrm{Cl}^{-}\right]$. Red line reprents the best fit of the data to a $1: 1$ binding mode
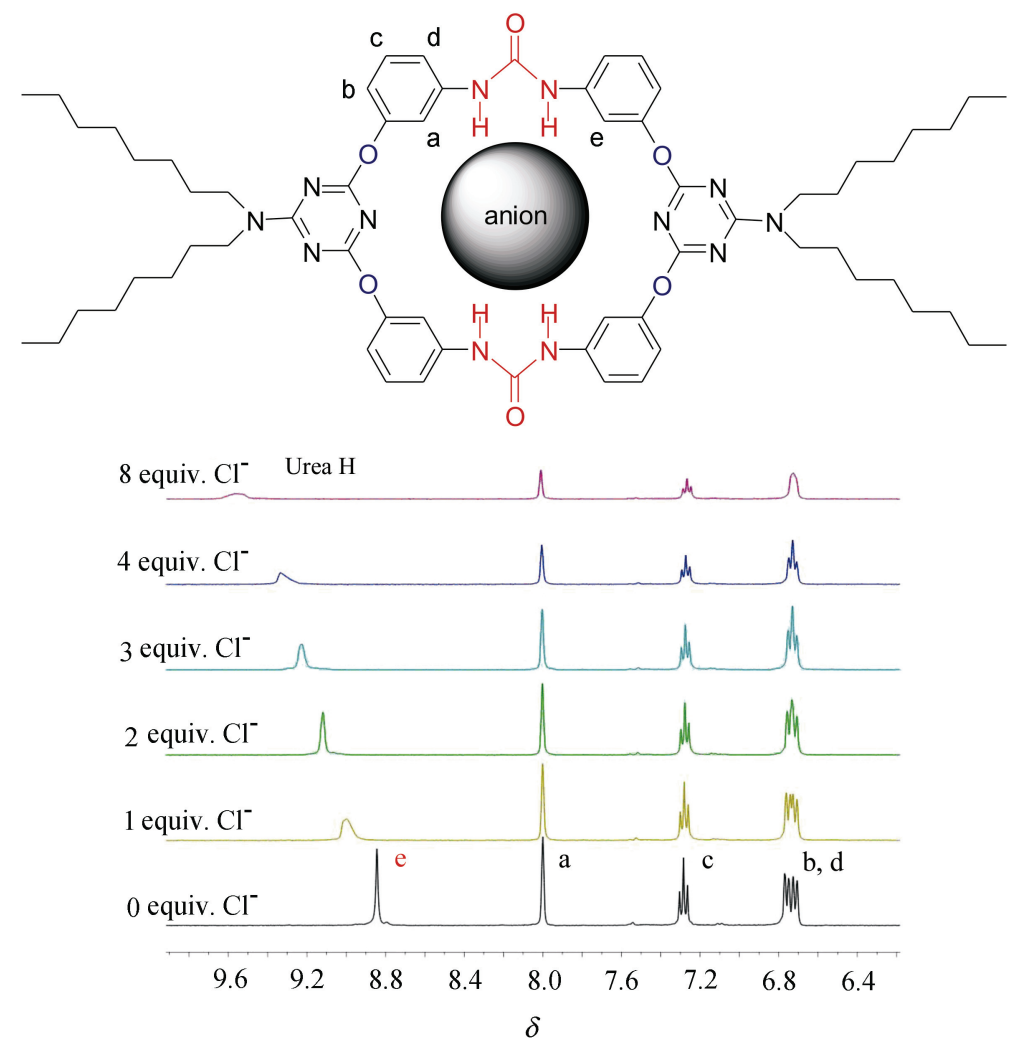

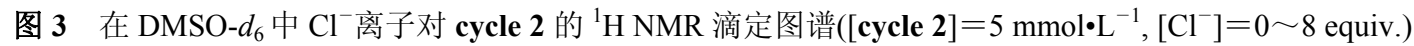

Figure 3 Partial ${ }^{1} \mathrm{H}$ NMR spectra of $5 \mathrm{mmol} \cdot \mathrm{L}^{-1}$ cycle 2 in DMSO- $d_{6}$ recorded upon titration with $n$ - $\mathrm{Bu}_{4} \mathrm{NCl}(0 \sim 8$ equiv.)

团上氢 $\left(\mathrm{H}_{\mathrm{e}}\right)$ 的化学位移在 $\delta 8.84$, 随着 $\mathrm{Cl}^{-}$的加入, 艮基 团上的 $\mathrm{H}_{\mathrm{e}}$ 信号峰逐渐向低场位移并伴随着峰形的变宽, 当 $\mathrm{Cl}^{-}$的浓度达到 cycle 2 的 8 倍时, $\mathrm{H}_{\mathrm{e}}$ 的化学位移向低 场位移了 $\delta 0.72$, 而其它氢的化学位移变化不大, 说明 环中的嫝基团是与阴离子相互作用的主要结合位点; 核 磁滴定过程中脲基团上活性氢始终表现为单峰, 表明环
上两个腿基团所处化学环境相同，即两个脲基团等效协 同作用于卤素阴离子. 除了脲基团上氢化学位移明显变 化外，随着 $\mathrm{Cl}^{-}$离子的加入，苯环上的质子 $\mathrm{H}_{\mathrm{a}}, \mathrm{H}_{\mathrm{b}}, \mathrm{H}_{\mathrm{c}}$, $\mathrm{H}_{\mathrm{d}}$ 化学位移也有轻微移动, $\mathrm{H}_{\mathrm{b}}$ 和 $\mathrm{H}_{\mathrm{d}}$ 的两组双重峰随着 $\mathrm{Cl}^{-}$的加入合并成 1 个三重峰, 分析可能是因为腿基团 与 $\mathrm{Cl}^{-}$离子之间的氢键对嫝基团的吸电子效应产生影 
响, 改变了苯环上质子的化学位移. 在大量 $\mathrm{Cl}^{-}$离子加

入后(8 equiv.), 核磁信号分辨率降低.

吸收光谱和核磁滴定实验结果表明, 大环与卤素阴 离子以 $1: 1$ 的模型结合, 脲基团是大环与卤素阴离子 作用的位点, 两个嫝基团等效协同作用于卤素应该是大 环与卤素阴离子之间具有较大结合常数的主要原因.

\section{3 结论}

利用羰基二咪唑和氨基高效合成嫝基团的反应以 及三聚氯氰分步取代反应的特点, 通过片段偶联法方便 地合成骨架中含有双脲基团氧杂大环化合物, 得到的大 环化合物可与卤素阴离子以 $1: 1$ 模型结合, 结合常数 达到 $10^{5} \sim 10^{8} \mathrm{~L} \cdot \mathrm{mol}^{-1}$, 结合强度顺序为: $\mathrm{F}^{-}>\mathrm{Cl}^{-}>$ $\mathrm{Br}^{-}>\mathrm{I}^{-}$. 这些研究结果为新型阴离子化学传感器的设 计合成以及大环化合物在离子响应材料方面的应用提 供了基础.

\section{References}

[1] (a) Martinez-Manez, R.; Sancenon, F. Chem. Rev. 2003, 103, 4419. (b) Schmidtchen, F. P.; Berger, M. Chem. Rev. 1997, 97, 1609. (c) Caltagirone, C.; Gale, P. A. Chem. Soc. Rev. 2009, 38, 520.

[2] (a) Ghadiri, M. R.; Granja, J. R.; Milligan, R. A.; Mcree, D. E.; Khazanovich, N. Nature 1993, 366, 324.

(b) Shimizu, L. S.; Hughes, A. D.; Smith, M. D.; Davis, M. J.; Zhang, B. P.; zur Loye, H. C.; Shimizu, K. D. J. Am. Chem. Soc. 2003, 125, 14972. (c) Shimizu, L. S.; Smith, M. D.; Hughes, A. D.; Shimizu, K. D. Chem. Commun. 2001, 1592.

[3] (a) Lagona, J.; Mukhopadhyay, P.; Chakrabarti, S.; Isaacs, L. Angew. Chem., Int. Ed. 2005, 44, 4844.

(b) Lankshear, M. D.; Beer, P. D. Acc. Chem. Res. 2007, 40, 657.

[4] (a) Custelcean, R.; Remy, P.; Bonnesen, P. V.; Jiang, D. E.; Moyer, B. A. Angew. Chem., Int. Ed. 2008, 47, 1866.

(b) dos Santos, C. L. M. G.; McCabe, T.; Watson, G. W.; Kruger, P. E.; Gunnlaugsson, T. J. Org. Chem. 2008, 73, 9235.

(c) Perez-Casas, C.; Yatsimirsky, A. K. J. Org. Chem. 2008, 73, 2275.

(d) Boiocchi, M.; Del Boca, L.; Gómez, D. E.; Fabbrizzi, L.; Licchelli, M.; Monzani, E. J. Am. Chem. Soc. 2004, 126, 16507.

(e) Hay, B. P.; Firman, T. K.; Moyer, B. A. J. Am. Chem. Soc. 2005, 127, 1810 .

(f) Lee, D. H.; Lee, K. H.; Hong, J. I. Org. Lett. 2001, 3, 5.

[5] (a) Ranganathan, D. Acc. Chem. Res. 2001, 34, 919.

(b) Brooks, S. L.; Garcia-Garrido, S. E.; Light, M. E.; Cole, P. A.; Gale, P. A. Chem. Eur. J. 2007, 13, 3320.

[6] (a) Snellink-Ruel, B. H. M.; Antonisse, M. M. G.; Engbersen, J. F. J.; Timmerman, P.; Reinhoudt, D. N. Eur. J. Org. Chem. 2000, 165. (b) Meshcheryakov, D.; Bohmer, V.; Bolte, M.; Hubscher-Bruder, V.; Arnaud-Neu, F. Chem. Eur. J. 2009, 15, 4811.

(c) Ranganathan, D.; Lakshmi, C.; Karle, I. L. J. Am. Chem. Soc. 1999, 121, 6103 .

[7] (a) Mooibroek, T. J.; Gamez, P. Inorg. Chim. Acta 2007, 360, 381. (b) Lowik, D. W. P. M.; Lowe, C. R. Eur. J. Org. Chem. 2001, 2825.

(c) Yang, X. P.; Lowe, C. R. Tetrahedron Lett. 2003, 44, 1359.

(d) Wang, M. X.; Yang, H. B. J. Am. Chem. Soc. 2004, 126, 15412.

(e) Wang, D. X.; Zheng, Q. Y.; Wang, Q. Q.; Wang, M. X. Angew. Chem., Int. Ed. 2008, 47, 7485 . 